\title{
Visual half field experiments are a good measure of cerebral language dominance if used properly: evidence from fMRI
}

\author{
Zoë R. Hunter ${ }^{1} \&$ Marc Brysbaert ${ }^{1}$ \\ ${ }^{1}$ Department of Psychology, Royal Holloway University of London, UK \\ Version: July $03^{\text {rd }}, 2007$ \\ for submission to \\ Neuropsychologia \\ (Category: Article)
}

Address correspondence to:

Zoë R. Hunter

Department of Psychology

Royal Holloway University of London

Egham, Surrey, TW20 0EX, United Kingdom

phone: +441784 414635

email: zoe.hunter@rhul.ac.uk

Short title: Assessing cerebral language dominance

Word count (Abstract): 194

Word count (complete doc.): 7984

4 Figures

2 Tables

1 Appendix 
Traditional neuropsychology employs visual half field (VHF) experiments to assess cerebral language dominance. This approach is based on the assumption that left cerebral dominance for language leads to faster and more accurate recognition of words in the right visual half-field (RVF) than in the left visual half-field (LVF) during tachistoscopic presentation. Information in the RVF is directly projected to the left hemisphere, whereas information presented in the LVF needs interhemispheric transfer to reach the left half of the brain. This interpretation of the RVF superiority for word recognition lacks direct evidence however, and a multitude of studies have lead to contradictory findings. To investigate this matter further we try to establish the ideal parameters for VHF experiments to measure language dominance, and subsequently compare laterality indices (LIs) obtained from RT patterns in bilateral VHF tasks to those LIs acquired in the same individuals during a mental word generation task in the fMRI scanner. Our results reveal a direct link between VHF advantages and individual language lateralization. Differences in behavioral performance between left-hemisphere dominant and right-hemisphere dominant individuals suggest that carefully designed VHF tests can be used as a reliable predictor of cerebral language dominance.

Key words: language; hemispheric asymmetry; interhemispheric transfer; left-handers; dichotic listening 


\section{Introduction}

In the past, intracarotid amobarbital anaesthesia, also known as the Wada test, was one of the most precise ways of determining the location of language areas prior to epileptic surgery (Wada \& Rasmussen, 1960). Today more modern, non-invasive techniques such as functional magnetic resonance imaging (fMRI) and functional transcranial Doppler sonography (fTCD) allow us to clearly identify which hemisphere is dominant for language production, revealing variations in hemispheric dominance patterns very much in line with what is known on the basis of the Wada test and the neuropsychological literature of brain lesions (Knecht et al., 1998; 2003).

Much more laterality research has been conducted on a behavioral level, however, making extensive use of visual half-field (VHF) tasks and dichotic listening tasks as the main methods of investigation.

In the VHF task stimuli are presented tachistoscopically in the left or the right parafovea and researchers measure the participants' speed and accuracy as a function of the presentation location. This technique is based on the crossing of the nasal optic fibers in the optic chiasm, which means that information displayed in the right visual half-field (RVF) is initially projected to the left cerebral hemisphere (LH), whereas information displayed in the left visual half-field (LVF) is initially projected to the right hemisphere (RH; see Bourne, 2006, for a review of the technique). In the dichotic listening task different stimuli are presented simultaneously to the left and the right ear and researchers register the participants' performance towards the stimuli presented to each ear. Although there is a bilateral projection of auditory information to the cortex, the contralateral projections are stronger than the ipsilateral projections, 
so that the left ear has better communication with the $\mathrm{RH}$, whereas the right ear has preferential access to the LH (e.g., Blumstein, Goodglass, \& Tartter, 1975).

In behavioral studies the predictions are that people with LH language dominance will show a RVF advantage in a VHF task with verbal stimuli and a right ear advantage in a dichotic listening task with word stimuli, whereas people with RH language dominance will show the reverse advantages. Information arriving in the subdominant hemisphere either requires interhemispheric transfer to reach the dominant hemisphere (which leads to an efficiency loss) or is processed more slowly by the less specialized hemisphere in which it arrived.

In the following, we will first give some examples of the problems these behavioral measures face and go on to discuss in detail the parameters which are necessary for a successful VHF set-up.

\section{Problems for behavioral measures}

VHF tasks and dichotic listening tasks partly owe their popularity to the ease with which they can be run. All that is required are a computer, headphones, a microphone and a button box (or a keyboard). At the same time, however, there have been continuous doubts about the reliability and validity of these behavioral measures. For instance, in a meta-analytic review of the literature, Voyer (1998) noted that the reliability of VHF tasks was only .56 for verbal tasks and .28 for non-verbal tasks. Even worse, the VHF advantages obtained in participants correlated only at .26 with the ear advantages obtained in the same participants, casting doubts on the assumption that VHF-differences and ear-differences measure the same processes. 
With the advent of modern techniques that allow to measure brain activity more directly, new validation opportunities for VHF tests have become available. Again, however, the picture is not very encouraging. Krach, Chen, and Hartje (2006) compared the laterality indices (LIs) obtained from a VHF experiment to those from fTCD. The VHF experiment consisted of lexical decisions made towards abstract nouns presented in the LVF and RVF. On each trial either two different nouns were presented or one noun and one non-word with an arrow at the fixation location pointing to the left or to the right. Participants had to indicate manually whether the stimulus to which the arrow pointed was a word or a non-word. At the same time, the blood flow to their left and right cerebral hemispheres was measured using functional transcranial Doppler sonography. In addition, Krach et al. (2006) measured hemispheric differences in blood flow in a word generation task ("mentally generate as many words as possible that start with the letter _'). As a group participants showed the expected RVF advantage in the VHF task, but the individual VHF differences did not correlate with the LIs based on fTCD in the word generation task $(\mathrm{r}=.18, \mathrm{n}=58)$ or even with the fTCD LIs measured during the VHF task itself. A similar story was reported by Cai, Lavidor, Brysbaert, Paulignan, \& Nazir (under revision). They measured ERPs in left-handed participants during a VHF lexical decision task and were able to distinguish a group of LH-dominant and RH-dominant participants on the basis of the ERP data. However, when they looked at the accuracy of their participants, there was only a small difference between both groups. Whereas the LH dominant group showed the expected RVF advantage (93\% correct in RVF vs. $69 \%$ correct in LVF), no LVF advantage was observed for the RH dominant group (94\% correct in RVF vs. 91\% correct in LVF). 
Bethmann, Tempelmann, De Bleser, Scheich, and Brechmann (2007) compared LIs obtained in dichotic listening (DL) with those obtained in an fMRI study (30 participants of whom 26 were righthanded). In the DL experiment two rhyming German words were presented (one to the left ear and one to the right ear) and participants had to indicate which words had been presented out of four choices consisting of the target words and two rhyming foils. In the fMRI study, participants had to decide whether two visually presented words had the same meaning or were semantically unrelated. The LIs in the DL experiment varied from -4.73 (clear left ear advantage) to +6.14 (clear right ear advantage), with $54 \%$ of the right-handed participants showing a significant right ear advantage. The fMRI data revealed LH dominance in 26 participants, bilaterality in 1 participant, and $\mathrm{RH}$ dominance in 3 participants. Crucially, the correlation between the LIs of both tasks was low $(r=.38$, $\mathrm{n}=30$ ), making the authors conclude that DL is not a valid task to measure laterality at an individual level. A more positive picture was published by Fernandes and Smith (2000). They compared the LI in the same DL task as Bethmann et al. (English words, however) with results of a Wada test in 28 children with epilepsy. They observed that 18 of the 19 children with LH dominance had the expected right ear advantage and that the only child with RH dominance had the expected left ear dominance. The 8 children with bilateral language representation predominantly had a small right ear advantage.

All in all, the evidence for the validity of behavioral tasks as a measure of laterality does not look impressive, certainly not at the individual level (but looking slightly better when groups of participants are compared). In particular, the status of the VHF task seems to be weak. This agrees with the criticisms that have been raised against this task time and time again, starting from Heron (1957) who argued that 
VHF differences were caused by a left- or rightwards bias in attention allocation across the visual field and not by cerebral laterality. On the other hand, a close look at the VHF tasks that were used in the validation studies, suggests that many of them were suboptimal (see below). So, it could be that some of low correlations were due to bad testing rather than to the fact that VHF differences are an invalid measure of hemispheric asymmetries.

In the remainder of this article, we describe our attempts to find out whether it is possible to design a VHF-experiment that yields a reliable and valid measure of language dominance. We do so by first listing the considerations we took into account and then describing the studies we ran.

\section{Requirements of a good VHF task to assess language dominance}

There must be sufficient trials. Brysbaert and d'Ydewalle (1990) looked at the confidence intervals of VHF differences in individual participants and were astonished by their magnitude. This agrees with the low reliability of many VHF experiments (Voyer, 1998; see above). Many researchers seem to underestimate the enormous variability in reaction times (SDs of $300 \mathrm{~ms}$ are no exception) and the range of uncertainty around accuracy scores that are based on small numbers of observations. For instance, Krach et al. (2006) limited the number of trials in their VHF task to a total of only 80 word targets per participant.

A second reason why a VHF experiment must comprise a rather high number of observations is that we know little about practice effects in VHF advantages. According to Seibel (1963) any difference between the first trials and later trials could 
reflect a novelty effect towards the task, which is unstable and may subside after a sufficient number of practice trials.

The stimuli in LVF and RVF must be matched. Another precaution that must be taken if data are to be used at an individual level is that the VHF differences must not be contaminated by the stimuli presented in LVF and RVF. Usually, psychology researchers circumvent this problem by counterbalancing their stimuli over participants: Half the participants see certain words in the LVF and other words in the RVF, whereas the other half gets the reverse arrangement. This works well as long as the data are limited to group comparisons, but can have detrimental effects if the data are analyzed at an individual level (e.g., in correlation analyses). Hence, the words that will be presented in the LVF and RVF should be matched. In addition, if individual data are to be used it is recommended to present the stimuli both in LVF and in RVF, so that there is no confound between VHF and words used. (Incidentally, if LVF and RVF presentations are blocked, for instance by swapping the words from session 1 to session 2, this still can have major negative effects on the reliability of the VHF differences if the reliability is calculated on the basis of the split-half technique.) Whether it is a good idea to repeat Krach et al.'s (2006) approach by using only 20 different words that are first read aloud three times by the participants (so that they are familiar with the items) and are then repeated over and over again in the trials, is less clear. The fewer stimuli that are used and the more they are repeated, the more the study deals with episodic memory retrieval, as opposed to word recognition.

Bilateral presentation is better than unilateral presentation. In as series of studies Boles $(1987,1990,1994)$ observed that the VHF differences were bigger and 
more stable when, in each trial, two different stimuli were presented simultaneously in the LVF and RVF than when only one stimulus was presented either in the LVF or in the RVF. This finding has been repeated in a series of unpublished experiments by Brysbaert on word naming, picture naming, clock face reading, bar graph reading, and symmetry detection (see also Iacoboni \& Zaidel, 1996). One reason for the superiority of bilateral presentation may be that the LVF stimulus has to compete with the RVF stimulus, which is easier when the target arrives in the dominant hemisphere and the competitor in the non-dominant hemisphere than in the reverse situation. This is the same reasoning as behind the clearer ear advantage in dichotic listening than in monaural listening. Another reason for obtaining better results with bilateral presentation may be that the stimulus onset in unilateral presentation is a strong attention catcher, which may lead to express saccades. These are very rapid reflexive eye movements to a stimulus onset and can be initiated within a time period as short as $80 \mathrm{~ms}$ (Fischer \& Weber, 1993). Finally, as we will see below, bilateral presentation also allows presentation of stimuli for a longer time.

Stimulus presentation must not be too degraded. Since stimuli in a VHF task have to be presented tachistoscopically in parafoveal vision, the presentation quality is always suboptimal. Visual acuity drops sharply outside the fixation location (Anstis, 1974) and the stimulus can not be presented for too long, as otherwise participants will make an eye-saccade towards it. How bad the presentation quality is depends on the size of the stimulus, the eccentricity, and the presentation duration. In general, researchers limit the presentation duration to $150 \mathrm{~ms}$ in order to avoid eye movements towards the target (although there is evidence for faster express saccades; see above). There is also a consensus that stimuli should be presented at least 1 degree 
of visual angle outside the fixation location to ensure unilateral projection (e.g., Bourne, 2006, but see the discussion section below). Finally, if stimuli are small, they become more difficult to recognize. Although there has been a lot of discussion about the impact of the stimulus quality, there is evidence that the more degraded the stimulus displays become, the more the RH is involved (Christman, 1989; Sergent, 1983).

With respect to the presentation duration, a very interesting study was recently reported by Walker and McSorley (2006). They showed that when two stimuli appear simultaneously left and right of the fixation location with an arrow at the fixation location pointing towards the target, participants need more than $200 \mathrm{~ms}$ to initiate a voluntary saccade to the target (with a mean saccade onset latency of $340 \mathrm{~ms}$ ). This means that in bilateral displays with an arrow indicating the stimulus to be named, stimuli can be presented for up to $200 \mathrm{~ms}$.

In order to restrict presentation duration to the chosen time window, it has been advised by several authors to replace the stimulus by a patterned mask at the offset. This prevents iconic memory of the target and any possibility of afterglow effects on the screen.

The fixation location must be controlled. In VHF tasks participants are asked to fixate a fixation cue at the beginning of a trial. Shortly afterwards a stimulus is presented left and/or right of the fixation location and participants have to process the stimulus. They are allowed to move their eyes as soon as the stimulus appears, but not on beforehand. They are explicitly instructed about the importance of the initial central fixation, but from eye-tracking studies it has become clear that participants rarely fully comply with the instruction, unless central fixation is constantly 
monitored (Jordan, Patching, \& Milner, 1998). One way to monitor adequate fixation is with the use of an eye-tracker, but this interferes with the possibility of vocal responses (eye-trackers only yield reliable results as long as participants do not move too much). An alternative is to briefly flash important information at the fixation location together with the information presented in LVF and/or RVF. Such information is easy to insert in a design with bilateral stimulus presentation. By presenting a left- or rightwards pointing arrow indicating which stimulus has to be named, participants are forced to direct their attention towards the fixation location at the beginning of a trial, in order to give a correct response.

Language dominance is defined as the laterality of word production. If the left hemisphere is called the dominant hemisphere, it is because lesions in the frontal lobe of this hemisphere (i.e. Broca's area and the surrounding tissue) lead to speech output problems in the majority of people. Similarly, the Wada test mostly deals with speech production, as do the majority of tasks used to determine language dominance with fMRI and fTCD. The reason for this is that speech production is the most clearly lateralized function in the human brain (Kosslyn, 1987). Thus, an optimal VHF task involves word naming and not lexical decision. Very little is known about the control processes that are needed in lexical decision to translate the word/non-word decision into a manual response. In addition, relatively little is known about the laterality of the information upon which a lexical decision is based.

In languages read from left to right other factors than LH dominance are favoring RVF processing. In languages read from left to right, it is very easy to observe a RVF advantage with verbal stimuli, whereas it is extremely difficult to 
obtain a LVF advantage of the same size. This is only partly due to the fact that the vast majority of people are left dominant for language processing. Other variables that play a role are the reading direction (the RVF advantage is bigger for languages read from left to right, like English, than for languages read from right to left, like Hebrew) and the fact that more information is available at the word beginning than at the word end. These variables not only play a role in parafoveal word recognition (Efron, 1990), but also in foveal word recognition (see the discussion of the optimal viewing position below; Brysbaert \& Nazir, 2005). The reading direction and the information load distribution within words become more important as words grow longer, which is one of the reasons why the RVF advantage is larger for long words than for short words (see also Ellis, 2004). The implication for VHF studies is that the stimulus words must be short (3-5 letters). Otherwise it is difficult to observe any LVF advantages in languages such as English, German, French, or Dutch.

To circumvent the problem of left-right asymmetries in word processing, McKeever proposed the object naming task. This task involves the repeated naming of a small number of line drawings of objects presented in the LVF or RVF (e.g., drawings of an apple, a boat, or a house). Because the bottleneck is the selection of the appropriate name among the set of competitors (rather than the recognition of the simple pictures), the task is supposed to measure the laterality of speech production, which seems to be confirmed by finding a RVF advantage in over $90 \%$ of righthanders (e.g., McKeever, Seitz, Krutsch, \& Van Eys, 1995).

\section{Correlations between tasks are low when there is little variance in} performance. When scores of two tasks are correlated, it is important to take into account the full range of scores. Otherwise, chances are high that no correlation will 
be observed. For instance, although there is a strong positive correlation between verbal and mathematical abilities in the population as a whole, it is perfectly possible to observe a null-correlation (or even a negative correlation) among students in top universities. The reason for this is that only scores of the top 5-10\% of the population range are included, which all come from a narrow (high) range, where differences in scores are little influenced by the general intelligence of the students but by their interests (some have specialized more in literacy, others in mathematics).

Something similar may happen in validity studies of behavioral laterality measures, when researchers only look at one type of participants. A prime example may be the dichotic listening study by Bethmann et al. (2007), discussed above. In this study, the LIs of 26 right-handers and 4 left-handers were correlated. On beforehand, however, the authors could have known that they were unlikely to find more than 3 participants with non left language dominance (over $90 \%$ of the righthanders and over $70 \%$ of the left-handers are left dominant). If we look in more detail at their findings, we indeed observe that only three participants are classified as right language dominant on the basis of fMRI; however, we also see that these three participants were those with the strongest left ear advantage. So, if Bethmann et al. (2007) had included more left-handers, they probably would have found a stronger correlation between performance on the DL task and language dominance, as repeatedly reported in the neuropsychological literature (Fernandes \& Smith, 2000; Fernandes, Smith, Logan, Crawley, \& McAndrews, 2006; Hughdahl, Carlsson, Uvebrant, \& Lundervold, 1997).

\section{Designing a good VHF task: Does it all add up?}


In the previous part we have argued that it is not a good approach to correlate just some VHF task with fMRI or fTCD or ERP data, in order to test the validity of the VHF task to assess language dominance. What we have not done yet, however, is show that taking the various considerations into account suffices to come up with a good and valid VHF measure of language laterality. This is what we address in the empirical part below. In particular, we have designed a VHF task that:

- contained an acceptable number of observations (>150),

- made use of matched stimulus sets,

- comprised bilateral stimulus presentation,

- used stimuli that were clearly visible and masked at the offset,

- made use of adequate fixation control at the beginning of a trial,

- was based on stimulus naming,

- was tested on left-handed participants to increase the range of LIs,

- and was validated against an fMRI study.

Further, because of the concerns that have been raised about the influence of the reading direction on VHF differences, we decided to use two tasks: One based on the presentation and naming of words, and another based on the presentation and naming of symmetrical line drawings of objects.

To increase the probability of including people with atypical language dominance, we invited left-handed individuals only to take part in our study. Knecht et al. (2000) observed that in a student population up to $25 \%$ of strong left-handers have bilateral or right hemisphere dominant representation of their speech production, against a mere $5 \%$ of strong right-handers. It was a vital component of our study to be 
able to compare behavioral performance differences ranging from RVF advantage to LVF advantage with expected LH to RH dominance patterns obtained in the scanner.

\section{Experiment}

Participants initially completed two behavioral VHF tasks: a bilateral $V H F$ picture-naming task and a bilateral VHF word-naming task. Then, a subset of the participants took part in an fMRI based validation study. This study comprised the assessment of hemispheric dominance for language production, in a set-up employing a mental word generation task known to produce marked lateralization, in both fMRI and fTCD settings, which is in agreement with the lateralization determined by a Wada test (Knecht et al., 2000, 2001, 2003).

\section{Methods}

Procedure All participants gave informed consent and subsequently took part in two lines of research which were approved by the departmental ethics committee. All participants were left-handed, as assessed by a questionnaire based on the Edinburgh Handedness Inventory. The task to be performed in the scanner was practiced off-line prior to the onset of the experimental trials. All participants were native English speakers.

\section{Behavioral tasks}

A sample of 26 left-handed male and female students participated in the behavioral part of the experiment ( 7 male, 17 female; mean age 19.7). All experiments were programmed using DMDX (Forster \& Forster, 2003). The order of 
presentation of the words and pictures in the respective experiments was pseudorandomized using the DMDX scrambling option.

VHF picture-naming Participants viewed a monitor at a distance of $\sim 60 \mathrm{~cm}$ and were asked to fixate a cross in the centre of the screen (fixation-space) at the beginning of each trial. Participants initiated the onset of the experiment by pressing the spacebar on the keyboard. Five symmetrical line drawings (a house, tree, boat, lamp and book; all of which represent monosyllabic words; see Figure 1) were presented repeatedly in randomized order and stayed on screen for $200 \mathrm{~ms}$ each. Pictures were presented at a visual angle of $1.91^{\circ}$ from fixation (with the outer edge at $10.98^{\circ}$ ). Presentation occurred in a bilateral fashion, that is one picture was presented in the left visual halffield (LHF), while another was presented simultaneously in the right visual half-field (RHF). Bilateral presentation was controlled in such a way that no matching pictures were displayed at the same time. The picture to be named was indicated by an arrow that was flashed in the fixation space simultaneously with the bilaterally presented pictures. This ensured fixation of the fixation space throughout the experiment, since the arrow gave the cue which side to attend to in order to give a correct response. The arrow stayed on screen while the pictures were masked with a cluster of randomly oriented lines that were presented for $200 \mathrm{~ms}$ and matched the pictures in size. Each picture was shown and had to be named 16 times within the LVF and 16 times within the RVF. In all, participants named a total of 160 pictures. Responses were collected by means of a voice trigger, where the onset of speech was registered as reaction time for a specific stimulus. 
VHF word-naming The VHF word-naming task is adapted from Brysbaert (1994). Participants viewed a monitor at a distance of $\sim 60 \mathrm{~cm}$ and were asked to fixate a cross in the centre of the screen (fixation-space) at the beginning of each trial. Participants initiated the onset of the experiment by pressing the spacebar on the keyboard. 96 three- to six-letter words (a set of 24 words of each length; see the Appendix) were presented bilaterally in randomized order and stayed on screen for $200 \mathrm{~ms}$ each. Words were presented at a visual angle of $0.95^{\circ}$ to $2.39^{\circ}$ from fixation (with the outer edge at $3.82^{\circ}$ ). Each word was presented once within each VHF and also had to be attended to and named correctly once in each VHF, which was achieved via two experimental runs (List 1 and List 2). In all, participants named a total of 192 words. The words were adapted from Weekes (1997), and were controlled for frequency and neighborhood size. All words were matched for initial phoneme and letter to ensure that reaction time differences were not due to an unbalanced distribution of shorter or longer initial phonemes/letters. In addition, bilateral presentation occurred in such a manner that word stimuli were displayed simultaneously with a controlled pair word (see the Appendix) that would never start with the same phoneme/letter, to reduce the risk of mid-word error correction after initially attending to the wrong side. The word to be named was indicated via an arrow that was flashed in the fixation space simultaneously with the bilaterally presented words. The arrow stayed on screen while the words were masked with a sequence of ASCII codes 35 (\#) that were presented for $200 \mathrm{~ms}$ and had the same length as the preceding stimulus. Responses were collected by means of a voice trigger, where the onset of speech was registered as reaction time for a specific stimulus. 
LI calculations Timing errors (>1500 ms) and naming errors were eliminated from the picture-naming data (mean error rate $1.51 \%$ ) and the word-naming data (mean error rate $5.03 \%$ ). To analyze the data sets, mean reaction times were calculated for the RVF and LVF for both VHF tasks, resulting in a laterality index (LI) for each task and each individual (Table 1), which was derived through the formula:

$$
L I_{(V H F)}=\left(R T_{-} L V F\right)-\left(R T_{-} R V F\right)
$$

This information was used to determine each participants VHF advantage, with negative LI values signaling a LVF advantage and positive values representing a RVF advantage.

\section{fMRI task}

Participants From the original sample of 26 lefthanders, we selected ten participants for fMRI based assessment of cerebral dominance. We chose a subset of six individuals who showed a strong RVF advantage in the VHF tasks (out of 15 showing this advantage), two with no clear VHF difference (out of 7), and two who showed a clear LVF advantage (out of $4^{1}$ ).

Data acquisition All 10 participants were scanned (4 male, 6 female; mean age 19.8). We used a mental word generation task to assess hemispheric dominance in a $3 \mathrm{~T}$ Magnetom Trio scanner (Siemens, Germany) fitted with an eight-channel head array RF coil. Ten single letters with the highest beginning-of-word frequency were presented in randomized order in the activation blocks. Participants had to silently

\footnotetext{
${ }^{1}$ The third and fourth participants with strong LVF advantage would unfortunately not participate in the fMRI part of the study.
} 
generate as many words as possible starting with the displayed letter. In a control phase the meaningless letter string "dada" was presented and had to be repeated continuously. Each activation and control block lasted $18 \mathrm{~s}$, followed by an $18 \mathrm{~s}$ rest interval. The stimulus onset was synchronized with the scanner pulse for each activation block. Blood oxygen-level dependent changes were measured using gradient-echo echo planar $\mathrm{T} 2 *$-weighted imaging sequences. Whole brain volumes comprising 36 axial slices each were acquired every 3 s (TE 32, flip angle $90^{\circ}$, resolution $3 \times 3 \times 3$, matrix $64 \times 64$, slice thickness $3 \mathrm{~mm}$, bandwidth 1346). In all, 243 scanning volumes were obtained for each participant. In addition, high resolution anatomical images were acquired (TR 1830, TE 5.56, flip angle $11^{\circ}$, resolution $1 \times 1 x 1$, 256x256 image matrix, 160 sagittal slices).

Data analysis The data were analyzed with the SPM2 software package (available online http://www.fil.ion.ucl.ac.uk/spm/). Images were realigned to the first functional volume to correct for motion artifacts and normalized into standard Talairach-type space using an EPI template. To reduce effects of random noise normalized data were spatially smoothed using a Gaussian kernel (FWHM 6mm). In addition, to correct for autocorrelation and minimize bias in estimates of the standard error, temporal smoothing was performed by applying a high-pass filter to the time series with a cutoff period of $100 \mathrm{~s}$. For statistical analysis the general linear model was employed to map the hemodynamic response curve onto each experimental condition using boxcar regressors. The boxcar function was then fitted to the time series at each voxel resulting in a weighted $\beta$-image. The fitted model was converted to a $t$-statistic image which constitutes the statistical parametric map. Images for each individual were 
corrected for family wise error $(\mathrm{FWE})$ at $\mathrm{p}=0.05$. The minimum cluster size was set to 20 activated voxels.

LI calculations After pre-processing and statistical analysis of the scanning data, the degree of cerebral dominance was calculated for each participant regarding those voxels that were significantly more active in the activation than in the control phase. Levels of activation were compared between the left and right hemispheres in predefined anatomical regions of interest (ROI), which encompassed regions in the inferior frontal cortex in both hemispheres, including BA 44 and BA 45 (Table 2). Each laterality index (fMRI LI) was derived by the formula

$$
L I=\frac{A_{L}-A_{R}}{A_{L}+A_{R}}
$$

where $A_{L}$ refers to the number of activated voxels in the left ROI and $A_{R}$ to the number of activated voxels in the right ROI. Seven participants showed a positive LI, three participants had a negative LI. Those individuals with LI $>+0.4$ were classed as left-dominant (6), those with indices $-0.4>\mathrm{LI}<+0.4$ as bilateral (2), and individuals with LI $<-0.4$ as right-dominant $(2)$. Mean scan images were calculated for leftdominant and right-dominant groups for comparison with the mean VHF graphs for word- and picture-naming (Figures 2 and 3).

The above method for LI calculation has been applied successfully in several studies (e.g., Knecht et al., 2003; Rutten, Ramsey, van Rijen \& van Veelen, 2002) and can be used to reliably identify hemispheric dominance for language. There is a debate, however, regarding the reproducibility of the degree of laterality indicated in the LI values. We calculated LIs based on the extent of activation in the ROI. The 
degree of laterality expressed in the LIs is dependant on the chosen statistical threshold and should therefore be treated with caution. The approach itself, however, has been shown to produce LIs which are not significantly different from LIs calculated via other means and which correlate well with LIs calculated from Wada and fTCD measurements (e.g., Chelbus et al., 2007; Jansen et al., 2004). In our study, laterality for those participants who were strongly left dominant and those who were strongly right dominant was exceptionally clear, regardless of cut off p-values chosen.

\section{Results}

Based on the strength of the VHF advantages from the word-naming task and under comparison with the VHF advantages for the picture-naming task, 15 participants were judged to have a clear RVF advantage, seven showed no clear VHF advantage and four individuals showed a clear LVF advantage (Table 1). A correlation calculated between the LIs for word- and picture-naming was highly significant $(r=0.80, p<0.01, t=6.532)($ Figure 4$)$.

For the ten individuals who took part in the scanning experiment we went on to compare LIs from both VHF tasks to those determined through fMRI (Table 2). The VHF picture-naming LIs significantly correlated with the fMRI LIs $(r=0.77, \mathrm{p}<$ 0.01, $\mathrm{t}=3.382)$. Similarly, the VHF word-naming LIs and the fMRI LIs $(\mathrm{r}=0.63, \mathrm{p}<$ $0.1, \mathrm{t}=2.273$ ) revealed significant positive correlations. 


\section{Discussion}

We started this research to find out whether it is possible to use behavioral VHF data for the assessment of cerebral language dominance. The existing literature was not very promising, but we were struck by the lack of experimental rigor in many studies. Assessing language dominance with a VHF set-up has one important advantage: It is fast and cheap. For the present studies, all we needed was a computer with freely available software to measure RTs, a microphone, and some simple, straightforward statistical tools to analyze the data. In contrast, each fMRI scan cost us $£ 300$, required access to an fMRI scanner, and quite some extra knowledge about data gathering and analysis. Given the difference in investment, it became important for us to find out whether the scanning could be limited to a subgroup of participants who on the basis of the VHF data were likely to have an interesting laterality pattern.

Our findings were unexpectedly reassuring: Participants with a clear RVF advantage in the picture-naming and word-naming tasks, were shown to be left dominant in the scanner, whereas participants with no VHF advantage or a LVF advantage turned out to be bilateral or right dominant in the scanner (Table 2). In addition, the tasks yielded clear LVF advantages in the right dominant participants, relieving us from the necessity to interpret small RVF advantages as signs of right hemisphere dominance, as we had feared on the basis of Cai et al. (2007, see the introduction). Apparently, the use of clearly visible, short words together with bilateral presentation enables us to observe LVF advantages with verbal stimuli. There were no clear differences between the object naming and the word naming task and both can be used interchangeably (or in tandem, as we have done here). Indeed, the high correlation between both tasks (Figure 4) not only is a testimony of the 
similarity of their VHF asymmetries but also of the reliability of these measures in the individual tasks. Reducing confounding factors in our VHF design has proven beneficial for obtaining stable LIs and confirms that VHF naming tasks can be used to reliably predict cerebral dominance for language production. This is illustrated in Figure 2, where we present the VHF data of the 6 right dominant and 2 left dominant participants, as indicated by their fMRI profile (Figure 3).

We are not claiming that our VHF data are good enough for clinical purposes, given the existence of well-documented and validated imaging protocols. However, what we hope to have shown is that VHF experiments can be used as a prescreening device for experiments that look at differences in processing between left dominant and right dominant individuals. This makes it possible to do this type of research (either based on imaging or on other behavioral measures) in a much more focused and affordable way.

Although we have attested the usefulness of VHF experiments to measure cerebral dominance, we would like to finish by pointing out that in the mean time we have developed a second behavioral technique that looks even more promising (Hunter \& Brysbaert, in press; Hunter, Brysbaert, \& Knecht, 2007). This technique, called the optimal viewing position (OVP) technique, starts out from the assumption that it is not necessary to present words parafoveally in order to measure hemispheric differences. The parafoveal presentation of stimuli in LVF and RVF was thought to be necessary because researchers believed that stimuli presented in central vision were sent bilaterally to the LH and RH. Several reviews of the literature (Brysbaert, 1994, 2004; Lavidor \& Walsh, 2004; Shillcock, Ellison \& Monaghan, 2000) have indicated, however, that this assumption is likely to be false and that the fovea is split down the middle. This means that language asymmetry can be measured by comparing word 
naming latencies for words fixated on the first letter with naming latencies for words fixated on the last letter. There is even some evidence that the OVP measure is capable of making a distinction between participants with bilateral language representation and those with RH language representation, which was not clearly present in the current VHF tasks (Hunter \& Brysbaert, in press).

All in all, our study has shown that when researchers adhere to the same standards in VHF studies as in fMRI studies (using well-controlled stimuli and a protocol that is based on a careful consideration of previous findings) they can attain a similar level of precision. Suggestions of the contrary in the past are likely to be due to inconsistencies as far as the VHF tasks were concerned. For convenience, we include the stimulus materials that were used in the present experiments, so that other experimenters can make use of them.

Acknowledgements This work was supported by grants from the European Research and Training Network: Language and Brain (Marie-Curie Program). We thank N. Ramnani and A. Smith for initial advice on fMRI-related issues. 


\section{References}

Anstis, S.M. (1974). Letter: A chart demonstrating variations in acuity with retinal position. Vision Research, 14(7), 589-592.

Bethmann, A., Tempelmann, C., De Bleser, R., Scheich, H. \& Brechmann, A. (2006). Determining language laterality by fMRI and dichotic listening. Brain Research, $1133,145-157$.

Blumstein, S., Goodglass, H. \& Tartter, V. (1975). The reliability of ear advantage in dichotic listening. Brain and Language, 2, 226-236.

Boles, D.B. (1987). Reaction time asymmetry through bilateral versus unilateral stimulus presentation. Brain and Cognition, 6, 321-333.

Boles, D.B. (1990). What bilateral displays do. Brain and Cognition, 12, 205-228.

Boles, D.B. (1994). An experimental comparison of stimulus type, display type and input variable contributions to visual field asymmetry. Brain and Cognition, 24, 184-197.

Bourne, V.J. (2006). The divided visual field paradigm: methodological considerations. Laterality, 11(4), 373-393.

Brysbaert, M. (1994). Interhemispheric transfer and the processing of foveally presented stimuli. Behavioural Brain Research, 64(1-2), 151-161. 
Brysbaert, M. (2004). The importance of interhemispheric transfer for foveal vision: A factor that has been overlooked in theories of visual word recognition and object perception. Brain and Language, 88(3), 259-267.

Brysbaert, M. \& D’Ydewalle, G. (1990). Individual analysis of laterality data. Neuropsychologia, 28(9), 901-916.

Brysbaert, M., \& Nazir, T. (2005). Visual constraints in written word recognition: Evidence from the optimal viewing-position effect. Journal of Research in Reading, 28(3), 216-228.

Cai, Q., Lavidor, M., Brysbaert, M., Paulignan, Y., \& Nazir, T.A. (in press). Cerebral lateralization of speech production and lateralization of the posterior visual word processing system. Journal of Cognitive Neuroscience.

Chlebus, P., Mikl, M., Brázdil, M., Pažourková, Krupa, P. \& Rektor, I. (2007). fMRI evaluation of hemispheric language dominance using various methods of laterality index calculation. Experimental Brain Research, 179, 365-374.

Christman, S. (1989). Perceptual characteristics in visual laterality research. Brain and Cognition, 11, $238-257$.

Ellis, A.W. (2004). Length, formats, neighbours, hemispheres, and the processing of words presented laterally or at fixation. Brain and Language, 88, 355-366. 
Efron, R. (1990). The Decline and Fall of Hemispheric Specialization. New Jersey: Erlbaum.

Fernandes, M.A. \& Smith, M.L. (2000). Comparing the Fused Dichotic Words Test and the Intracarotid Amobarbital Procedure in children with epilepsy. Neuropsychologia, 38, 1216-1228.

Fernandes, M.A., Smith, M.L., Logan, W., Crawley, A. \& McAndrews, M.P. (2006). Determining language lateralization in children with epilepsy: Comparison of fMRI and dichotic listening. Brain and Language, 96, 106-114.

Fischer, B. \& Weber, H. (1993). Express saccades and visual attention. Behavioral and Brain Sciences, 16(3), 553-610.

Forster, K.I. \& Forster, J.C. (2003). DMDX: A Windows display program with millisecond accuracy. Behavior Research Methods, Instruments, \& Computers, $35,116-124$.

Heron, W. (1957). Perception as a function of retinal locus and attention. American Journal of Psychology, 70(1), 38-48.

Hugdahl, K., Carlsson, G., Uvebrant, P. \& Lundervold, A.J. (1997). Dichotic-listening performance and intracarotid injections of amobarbital in children and adolescents. Archives of Neurology, 54, 1494-1500. 
Hunter, Z.R. \& Brysbaert, M. (in press). Theoretical analysis of interhemispheric transfer costs in visual word recognition. Language and Cognitive Processes.

Hunter, Z.R., Brysbaert, M. \& Knecht, S. (2007). Foveal word reading requires interhemispheric communication. Journal of Cognitive Neuroscience, 19(8), 115.

Iacoboni, M. \& Zaidel, E. (1996). Hemispheric independence in word recognition: Evidence from unilateral and bilateral presentations. Brain and Language, 53, 121-140.

Jansen, A., Flöel, A., Deppe, M., van Randenborgh, J., Dräger, B, Kanowski, M. \& Knecht, S. (2004). Determining the hemispheric dominance of spatial attention: A comparison between fTCD and fMRI. Human Brain Mapping, 23, 168-180.

Jordan, T. R., Patching, G. R., \& Milner, A. D. (1998). Central fixations are inadequately controlled by instructions alone: Implications for studying cerebral asymmetry. Quarterly Journal of Experimental Psychology, 51A, 371-391.

Knecht, S., Deppe, M., Ebner, A., Henningsen, H., Huber, T., Jokeit, H. \& Ringelstein, E.-B. (1998). Noninvasive determination of language lateralization by functional transcranial doppler sonography : A comparison with the wada test. Stroke, 29(1), 82-86. 
Knecht, S., Dräger, B., Deppe, M., Bobe, L., Lohmann, H., Floel, A., Ringelstein, E.B. \& Henningsen, H. (2000). Handedness and hemispheric language dominance in healthy humans. Brain, 123, 2512-2518.

Knecht, S., Dräger, B., Floel, A., Lohmann, H., Breitenstein, C., \& Deppe, M., et al. (2001). Behavioural relevance of atypical language lateralization in healthy subjects. Brain, 124(8), 1657-1665.

Knecht, S., Jansen, A., Frank, A., van Randenborgh, J., Sommer, J., Kanowski, M. \& Heinze, H.J. (2003). How atypical is atypical language dominance? NeuroImage, $18,917-927$.

Kosslyn, S.M. (1987). Seeing and imagining in the cerebral hemispheres: A computational approach. Psychological Review, 94, 148-175.

Krach, S., Chen, L.M. \& Hartje, W. (2006). Comparison between visual half-field performance and cerebral blood flow changes as indicators of language dominance. Laterality, 11(2), 122-140.

Lavidor, M. \& Walsh, V. (2004). The nature of foveal representation. Nature Reviews Neuroscience, 5, 729-735.

McKeever, F.W., Seitz, K.S., Krutsch, A.J. \& Van Eys, P.L. (1995). On language laterality in normal dextrals and sinistrals: Results from the Bilateral Object Naming Latency Task. Neuropsychologia, 33(12), 1627-1635. 
Rutten, G.J.M., Ramsey, N.F., van Rijen, P.C. \& van Veelen, C.W.M. (2002). Reproducibility of fMRI-determined language lateralization in individual subjects. Brain and Language, 80, 421-437.

Seibel, R. (1963). Discrimination reaction time for a 1,023-alternative task. Journal of experimental Psychology, 66(3), 215-226.

Sergent, J. (1983). The role in the input in visual hemispheric asymmetries. Psychological Bulletin, 93, 481-512.

Shillcock, R., Ellison, T. M., \& Monaghan, P. (2000). Eye-fixation behavior, lexical storage, and visual word recognition in a split processing model. Psychological Review, 107(4), 824-851.

Voyer, D. (1998). On the reliability and validity of noninvasive laterality measures. Brain and Cognition, 36, 209-236.

Wada J. \& Rasmussen T. (1960). Intracarotid injection of sodium amytal for the lateralization of cerebral speech dominance. Journal of Neurosurgery, 17, 266282.

Walker, R. \& McSorley, E. (2006). The parallel programming of voluntary and reflexive saccades. Vision Research, 26, 2082 - 2093.

Weekes, B.S. (1997). Differential effects of number of letters on word and nonword naming latency. Quarterly Journal of Experimental Psychology, 50A(2), 439- 
456. 
Table 1 VHF advantages for word and picture naming

\begin{tabular}{|c|c|c|c|c|}
\hline & $\begin{array}{l}\text { VHF word } \\
\text { RT difference } \\
\text { LVF - RVF }\end{array}$ & $\begin{array}{l}\text { VHF picture } \\
\text { RT difference } \\
\text { LVF - RVF }\end{array}$ & $\begin{array}{c}\text { VHF } \\
\text { advantage }\end{array}$ & $\begin{array}{l}\text { Selected } \\
\text { for fMRI } \\
\text { scan }\end{array}$ \\
\hline Sub_22 & 172.2 & 73.5 & RVF adv & $x$ \\
\hline Sub_08 & 120.1 & 55.5 & RVF adv & \\
\hline Sub_20 & 67.5 & 38.3 & RVF adv & \\
\hline Sub_06 & 67.4 & 1.3 & RVF adv & \\
\hline Sub_24 & 64.6 & 40.6 & RVF adv & $x$ \\
\hline Sub_10 & 62.9 & 45.8 & RVF adv & $x$ \\
\hline Sub_05 & 55.8 & 39.3 & RVF adv & \\
\hline Sub_11 & 52.7 & 40 & RVF adv & \\
\hline Sub_19 & 51.7 & 21.1 & RVF adv & $x$ \\
\hline Sub_07 & 45.9 & 9.3 & RVF adv & $x$ \\
\hline Sub_15 & 45.3 & 21.4 & RVF adv & \\
\hline Sub_25 & 44.9 & 43.9 & RVF adv & \\
\hline Sub_17 & 35.3 & 41.5 & RVF adv & \\
\hline Sub_21 & 33.1 & 4.1 & RVF adv & \\
\hline Sub_26 & 30.2 & 35.9 & RVF adv & $x$ \\
\hline Sub_18 & 23.7 & -16.1 & No clear adv & \\
\hline Sub_02 & 3.8 & -11.4 & No clear adv & \\
\hline Sub_09 & 3.7 & 17.7 & No clear adv & \\
\hline Sub_04 & -11.3 & 17.9 & No clear adv & \\
\hline Sub_14 & -23.1 & -27.4 & LVF adv & $x$ \\
\hline Sub_23 & -4.0 & 15.8 & No clear adv & \\
\hline Sub_03 & -31 & -3.3 & No clear adv & $x$ \\
\hline Sub_12 & -32 & -29.4 & LVF adv & \\
\hline Sub_01 & -42.9 & -47 & LVF adv & \\
\hline Sub_16 & -45.6 & -44.8 & LVF adv & $\mathrm{x}$ \\
\hline Sub_13 & -72.9 & 1.1 & No clear adv & $x$ \\
\hline
\end{tabular}


Table 2 Laterality indices compared for ten individuals

\begin{tabular}{|c|c|c|c|c|c|c|}
\hline $\begin{array}{l}\text { Active } \\
\text { voxels } \\
\text { eft ROI }\end{array}$ & $\begin{array}{c}\text { MNI } \\
\text { coordinates } \\
x, y, z\end{array}$ & $\begin{array}{c}\text { Active } \\
\text { voxels } \\
\text { right } \mathrm{ROI}\end{array}$ & $\begin{array}{c}\text { MNI } \\
\text { coordinates } \\
x, y, z\end{array}$ & fMRI LI & $\begin{array}{l}\text { LI VHF- } \\
\text { Picture }\end{array}$ & $\begin{array}{l}\text { LI VHF- } \\
\text { Word }\end{array}$ \\
\hline
\end{tabular}

\begin{tabular}{|c|c|c|c|c|c|c|c|}
\hline Sub_22 & 9469 & $-52+13+22$ & 2213 & $+55+10+14$ & +0.62 & 73.5 & 172.2 \\
\hline Sub_24 & 7054 & $-50+17+26$ & $\begin{array}{c}1606 \\
137\end{array}$ & $\begin{array}{c}+56+19+29 \\
+45+18+9\end{array}$ & +0.603 & 40.6 & 64.6 \\
\hline Sub_10 & 5812 & $-52+15+25$ & 169 & $+49+16+5$ & +0.945 & 45.8 & 62.9 \\
\hline Sub_19 & 7021 & $-51+13+24$ & 110 & $+59+13+36$ & +0.97 & 21.1 & 51.7 \\
\hline Sub_07 & 8953 & $-51+16+22$ & $\begin{array}{l}128 \\
100\end{array}$ & $\begin{array}{l}+49+17+29 \\
+43+5+31\end{array}$ & +0.95 & 9.3 & 45.9 \\
\hline Sub_26 & 11378 & $-51+15+24$ & 0 & & +1.00 & 35.9 & 30.2 \\
\hline Sub_14 & 143 & $-44+3+31$ & 8250 & $+52+16+22$ & -0.966 & -27.4 & -23.1 \\
\hline Sub_03 & 5265 & $-49+12+27$ & 2399 & $+59+11+17$ & +0.37 & -3.3 & -31 \\
\hline Sub_16 & $\begin{array}{l}1076 \\
238 \\
144\end{array}$ & $\begin{array}{l}-52+14+12 \\
-42+11+31 \\
-51+12+44\end{array}$ & 7206 & $+52+17+22$ & -0.66 & -44.8 & -45.6 \\
\hline Sub_13 & 2940 & $-50+10+22$ & $\begin{array}{c}3029 \\
1214 \\
100\end{array}$ & $\begin{array}{l}+54+15+10 \\
+51+7+32 \\
+54+33+2\end{array}$ & -0.19 & 1.1 & -72.9 \\
\hline
\end{tabular}


Figure 1 Stimulus material.
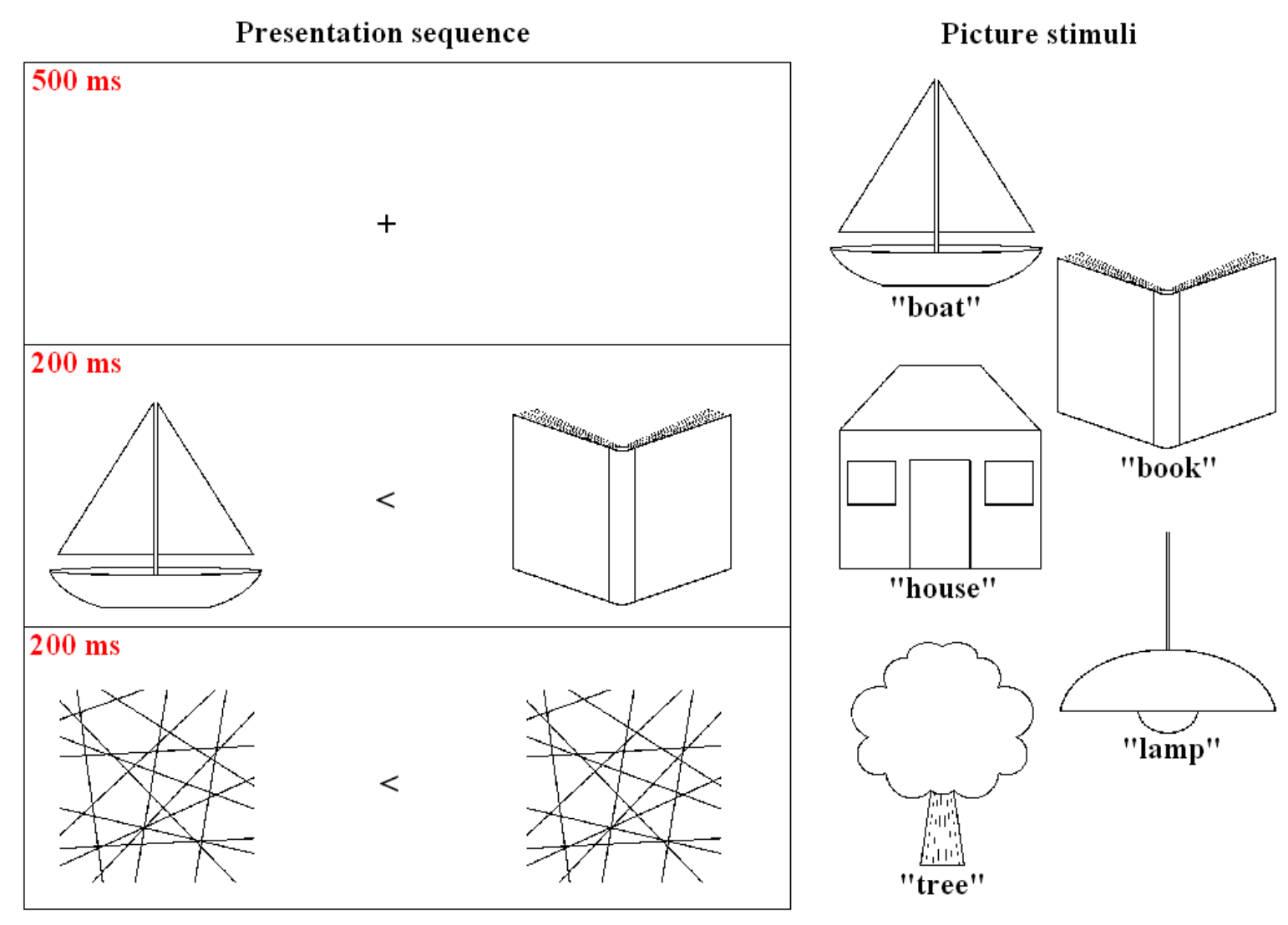

Figure 1 Stimulus material. An example sequence of the VHF picture-naming task is given (VHF word-naming trials looked similar). We used a set of five symmetrical line drawings, which had to be named repeatedly in the task. 
Figure 2 VHF graphs for typical and atypical dominance groups.

\section{VHF word naming}

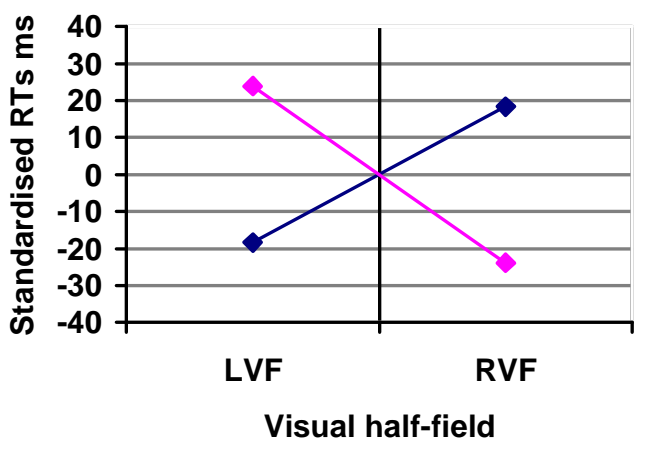

$\multimap$ atypical $\multimap$ typical
VHF picture naming

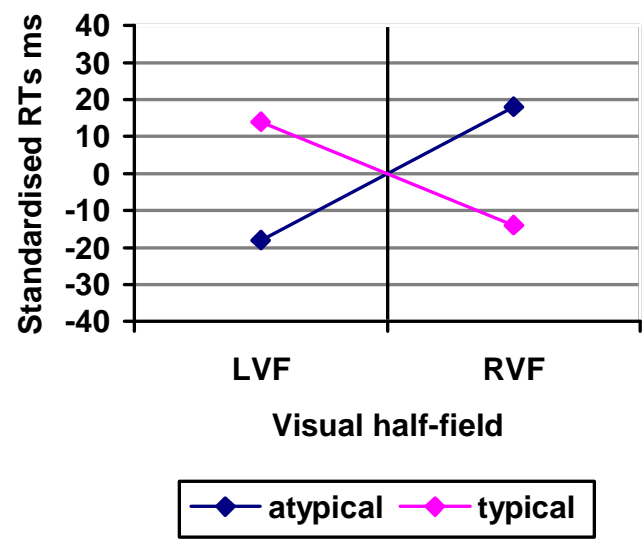

Figure 2 VHF graphs for typical (6) and atypical (2) dominance groups. Mean group reaction times for the subset of participants whose language dominance has been assessed in the scanner. Due to the small sample reaction times have been standardized for better comparison. There is a clear reversal of visual halffield advantages towards the LVF for the atypical language dominant group. 
Figure 3 Language lateralization in typical and a typical dominance groups.

a)

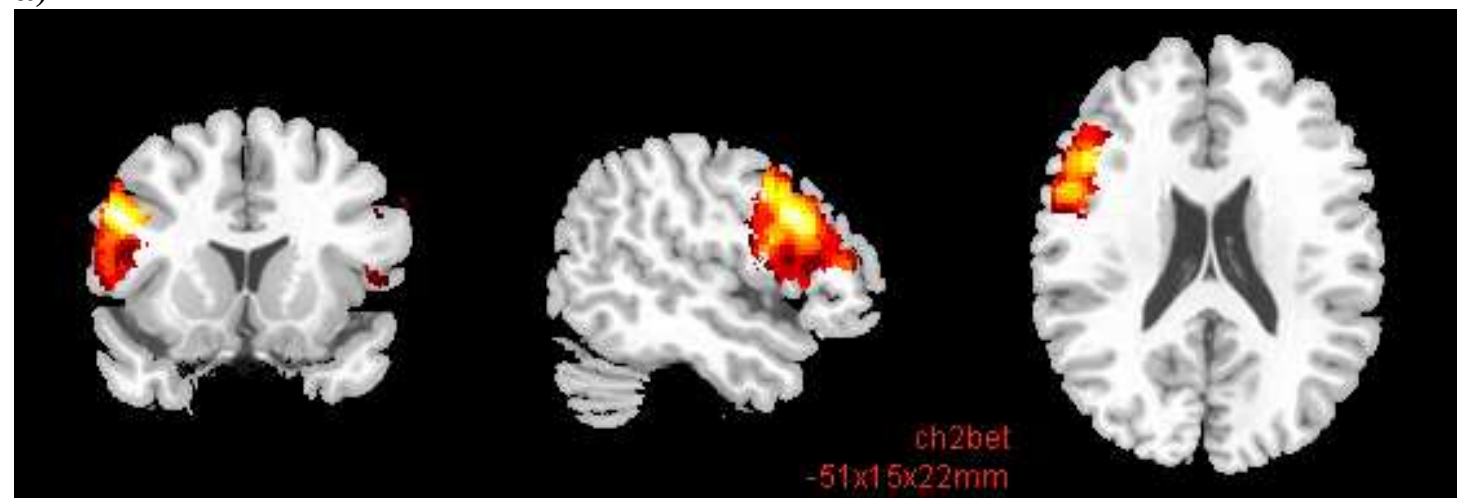

b)

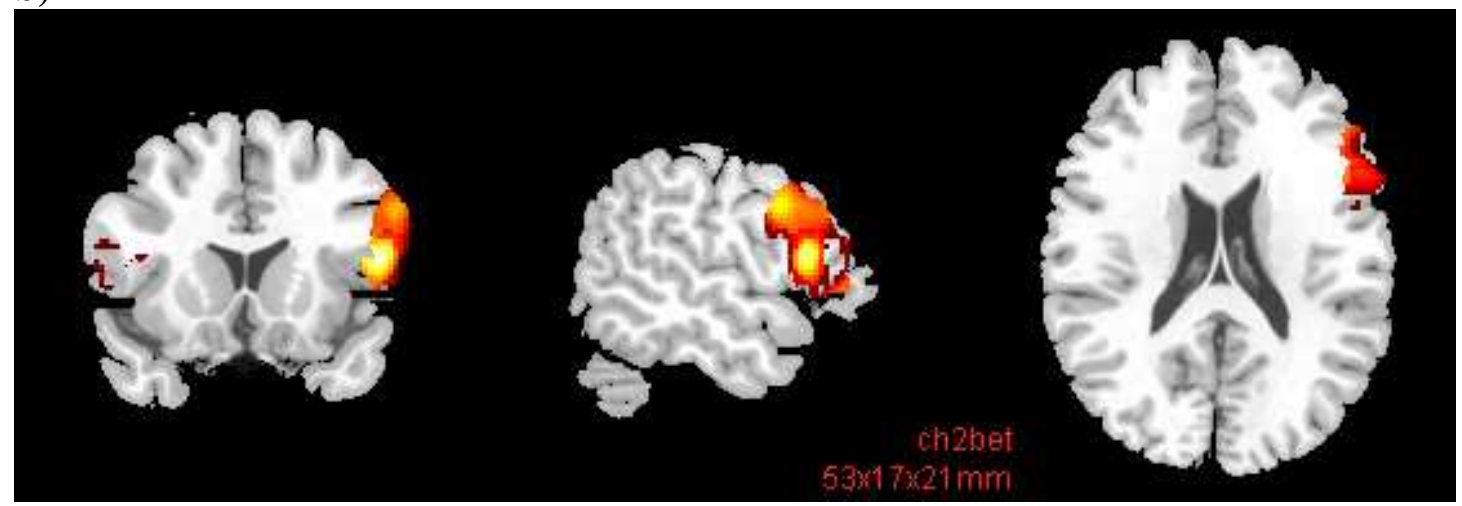

Figure 3 Language lateralization in typical and atypical dominance groups. a) Mean image in ROI for the four individuals with strongest left hemisphere dominance (coronal, sagittal and axial slices at MNI coordinates: $\mathrm{x}=-51, \mathrm{y}=$ $+15, \mathrm{z}=+22)$. b) Mean image in ROI for the two individuals with strongest right hemisphere dominance (coronal, sagittal and axial slices at MNI coordinates: $\mathrm{x}=53, \mathrm{y}=+17, \mathrm{z}=+21$ ). 
Figure 4 Scatter plot: LIs for VHF word- and picture-naming tasks.

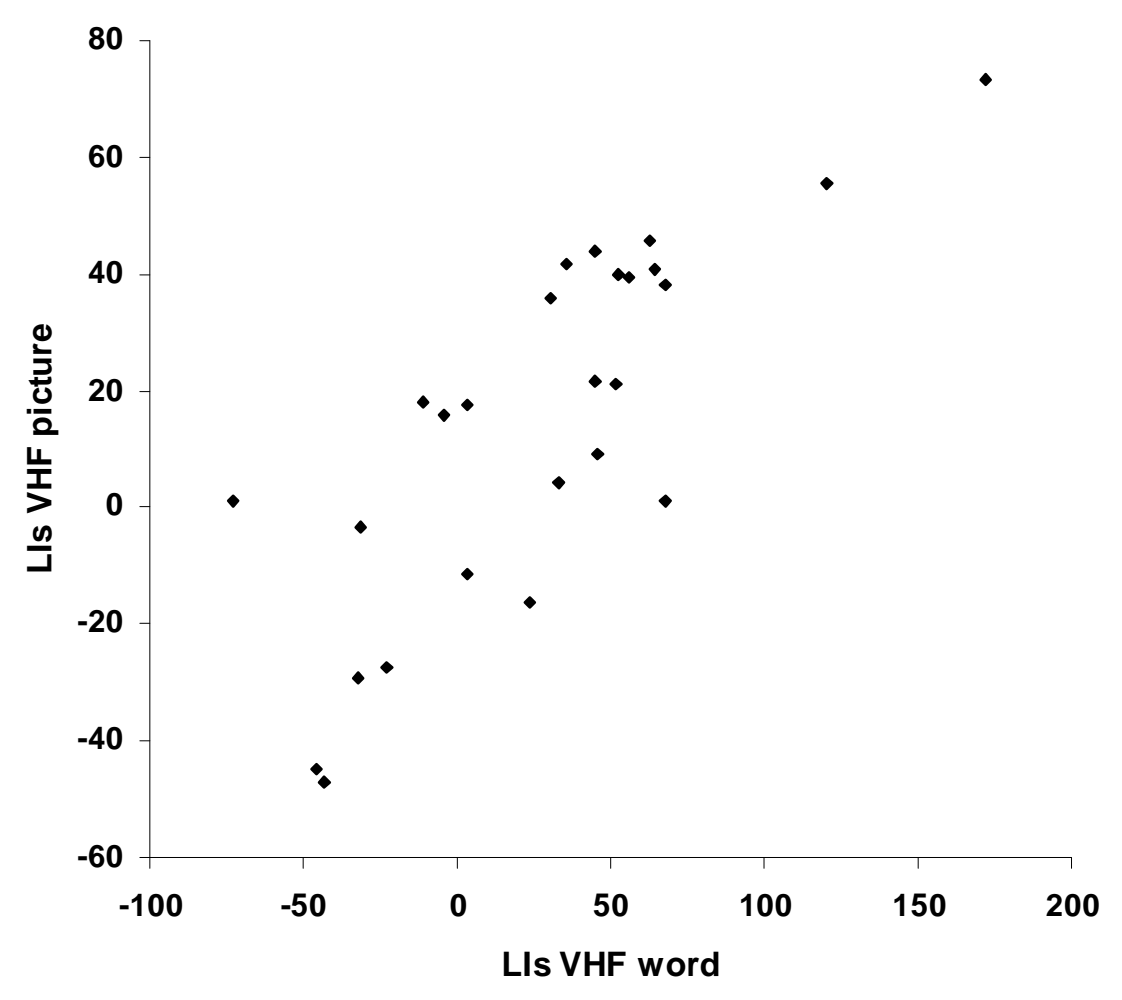

Figure 4 Scatter plot: LIs for VHF word- and picture-naming tasks. The VHF word-naming data show a strong positive correlation with the VHF picturenaming data $(\mathrm{r}=0.80, \mathrm{p}<0.01, \mathrm{t}=6.532)$. 
Appendix

\begin{tabular}{ccrr}
\hline 3-letter words & 4-letter words & 5-letter words & 6-letter words \\
$\begin{array}{c}\text { Stimulus Controlled } \\
\text { pair word }\end{array}$ & $\begin{array}{c}\text { Stimulus Controlled } \\
\text { pair word }\end{array}$ & $\begin{array}{r}\text { Stimulus } \\
\text { Controlled } \\
\text { pair word }\end{array}$ & $\begin{array}{c}\text { Stimulus } \\
\text { Controlled } \\
\text { pair word }\end{array}$
\end{tabular}

\begin{tabular}{|c|c|c|c|c|c|c|c|}
\hline bed & wig & bend & wisp & blame & whisk & boards & whence \\
\hline bee & toy & bill & toss & brawn & trump & branch & trance \\
\hline box & tho & blew & thug & bring & thorn & bridge & throng \\
\hline car & spy & care & suck & catch & sweep & called & swerve \\
\hline $\cot$ & spa & corn & stew & close & stack & claims & starch \\
\hline cry & sob & cost & spin & coast & speck & closed & sparse \\
\hline cut & sly & crew & snob & cross & sneer & crimes & snatch \\
\hline far & sip & film & slug & flesh & sleek & fought & sludge \\
\hline fig & sap & free & skid & fresh & skate & french & shrimp \\
\hline got & sag & grew & shod & grand & shrug & glance & scotch \\
\hline gun & pry & grey & prim & green & prank & grants & plunge \\
\hline hit & lop & home & loin & hence & latch & helped & planks \\
\hline let & ply & land & plum & large & plume & length & loaned \\
\hline pay & hob & plus & hock & plane & hoist & plants & hoarse \\
\hline per & gut & pope & grub & prime & grunt & prince & grudge \\
\hline sat & gig & salt & grid & scene & growl & screen & graves \\
\hline saw & fry & seem & fuse & sense & frock & served & freeze \\
\hline set & fox & seen & fray & sleep & flake & speech & flamed \\
\hline $\sin$ & cub & size & cult & smell & crust & sports & crunch \\
\hline sir & $\cot$ & slog & crab & snort & cramp & spring & corpse \\
\hline sky & cod & song & cord & space & click & strain & cloves \\
\hline sun & cad & stop & cave & store & carve & stress & clinch \\
\hline ten & bin & trip & bolt & trend & boast & twelve & bruise \\
\hline wet & beg & west & bled & while & blest & wheels & bronze \\
\hline
\end{tabular}

